\title{
Si Interlayer Thickness Dependence of Hysteresis Loop in Co/Si/Co/GaAs(001)
}

\author{
J. Islam* , Y. Yamamoto, E. Shikoh, A. Fujiwara, and H. Hori \\ School of Materials Science, Japan Advanced Institute of Science and Technology, 1-1 Asahidai, \\ Nomi, Ishikawa 923-1292, Japan
}

Received 5 June 2012, accepted in revised form 14 August 2012

\begin{abstract}
Magnetic hysteresis loop changes from two-phase to single-phase with decreasing $\mathrm{Si}$ interlayer thickness in $\mathrm{Co} / \mathrm{Si} / \mathrm{Co} / \mathrm{GaAs}$. Coercive field of $50 \mathrm{~nm}$ Co deposited on Si layer decreases with the increase of Si interlayer thickness. Deposition of Au layer between Co and $\mathrm{Si}$ changes the magnetic hysteresis loop. We propose that the formation of cobalt silicides at the interface of $\mathrm{Co}$ and Si modulate magnetic properties of the trilayer without Au buffer layer.
\end{abstract}

Keywords: Magnetic hysteresis; Interlayer; Co/Si/Co trilayer; Spins reversal; Two-phase hysteresis loop; Buffer layer.

(c) 2012 JSR Publications. ISSN: 2070-0237 (Print); 2070-0245 (Online). All rights reserved. doi: http://dx.doi.org/10.3329/jsr.v4i3.10852 J. Sci. Res. 4 (3), 561-567 (2012)

\section{Introduction}

For a long time, the spins of electrons have been ignored in the mainstream semiconductor electronics. The use of the spin degree of freedom of the electron in addition to its charge in spintronics device promises additional functionalities, increased data processing speed and integration densities, as well as reduced power consumption [1, 2]. The study of ferromagnetic thin film structures and their interfaces with semiconductor substrates has received much attention due to their potential technological applications as non-volatile magnetic memories, and in the field of spintronics [3-8]. The multilayers of ferromagnetsemiconductor (FS) hybrid structures have been the object of great recent interest. Multilayer of FS hybrid structure is of particular interest due to strong antiferromagentic coupling which is significance in applications using artificial antiferromagnets and ferromagnets as, for instance, in magnetic sensors [9] and in antiferromagnetically

\footnotetext{
*Corresponding author: mdjohurul@gmail.com, Present address: Department of Applied Physics and Electronic Engineering, University of Rajshahi, Rajshahi 6205, Bangladesh ( johurul@ru.ac.bd).
} 
coupled storage media for hard disk drive [10]. However, in the absence of an appropriate buffer layer [11] a significant chemical intermixing occurs between the transition metal and the semiconductor substrate which influences both the magnetization and anisotropy of multilayer. It is reported that the magnetic coupling between two ferromagnetic layers through amorphous Si interlayer (spacer) depends on the thickness of the Si spacer layer [12]. They found that the ferromagnetic layers are exchange coupled for Si spacer layer thicknesses equal to or less that $4 \mathrm{~nm}$. Antiferromagnetic coupling between ferromagnetic layers is observed in the Si thickness range 1.4-2.2 nm. The magnetic properties of $\mathrm{Co} / \mathrm{Ge} / \mathrm{Co}$ hybrid structure depend on temperature: the magnetization in low magnetic field remains practically zero up to a critical temperature $\left(T_{c}\right)$ and above $T_{c}$ it increases sharply towards saturation [8]. The magnetic properties of FS multilayer are not only affected by the thickness of Si interlayer but also the thickness of ferromagnetic layers itself. The thickness of ferromagnetic Fe films affects the magnitude of the interlayer exchange interaction through nonmagnetic Si interlayer [13]. Haque et al. [14] investigated the magnetic properties of $\mathrm{Ni} / \mathrm{Si} / \mathrm{Ni} / \mathrm{GaAs}$ multilayer. Two-phase hysteresis loop is observed in the multilayer. They also observed antiparallel spin state below $250 \mathrm{~K}$ which became the strongest at the lowest $(1.8 \mathrm{~K})$ temperature. It is well known that the carrier concentration of semiconductor layer can be controlled precisely. Magnetic properties of FS multilayer can be modulated by the transport properties of semiconductor spacer layer $[4,6,13]$. In this paper, we report the effect of Si-spacer layer and buffer layer on magnetic properties of the multilayer.

\section{Experimental details}

Substrates were chemically etched by semiconductor grade organic compound (Semico23-clean) and rinsed with deionized water before depositing Co thin films. The GaAs(001) substrate was Si-doped, with an electron carrier concentration of $1 \times 10^{24} \mathrm{~m}^{-3}$. Co films were deposited at a rate of $1.5 \AA^{-1}$ onto $\mathrm{GaAs}(001)$ substrate by e-beam evaporation method at a base pressure of $1 \times 10^{-6}$ Torr. On the other hand, the deposition rate of Si was only $0.3 \AA^{-1}$. The substrate was kept at room temperature during the film deposition. The structure of the multilayer became $\operatorname{Co}(50 \mathrm{~nm}) / \mathrm{Si}(\mathrm{d} \mathrm{nm}) / \operatorname{Co}(125 \mathrm{~nm}) / \mathrm{GaAs}$, where ' $d$ ' is the thickness of Si-spacer layer. The value of varies from $50 \mathrm{~nm}$ to $70 \mathrm{~nm}$. It is reported that the coercivities of $50 \mathrm{~nm}$ Co on Si and $125 \mathrm{~nm}$ Co on GaAs were 10 Oe and 120 Oe, respectively [15]. Therefore, $50 \mathrm{~nm}$ Co layer behaves as a soft magnetic layer and $125 \mathrm{~nm}$ Co layer behaves as a hard magnetic layer in the multilayer. The film thickness was measured by a quartz crystal oscillator, which was calibrated by an atomic force microscope. In X-ray diffraction measurement, the Co films grown on n-GaAs(001) substrate exhibited polycrystalline hcp structure. The magnetization was measured with a Quantum-Design MPMS after cooling the sample to the desired temperature. The magnetic field was applied parallel to the film plane, which was [001] crystallographic direction of the substrate. 


\section{Results and Discussion}

Dissimilar coercivities were observed in $\mathrm{Co} / \mathrm{GaAs}(001)$ and $\mathrm{Co} / \mathrm{Si}(001)$ bilayers [15]. A semiconducting, nonmagnetic $\mathrm{Si}$ is sandwiched between ferromagnetic Co layers to fabricate a multilayer of $\mathrm{Co} / \mathrm{Si} / \mathrm{Co} / \mathrm{GaAs}$ to achieve an antiparallel spin state in it. Fig. 1(a) shows the magnetization process of the multilayer having Si interlayer thicknesses $\left(d_{S i}\right)$ of 10 and $50 \mathrm{~nm}$, observed at $4.2 \mathrm{~K}$.

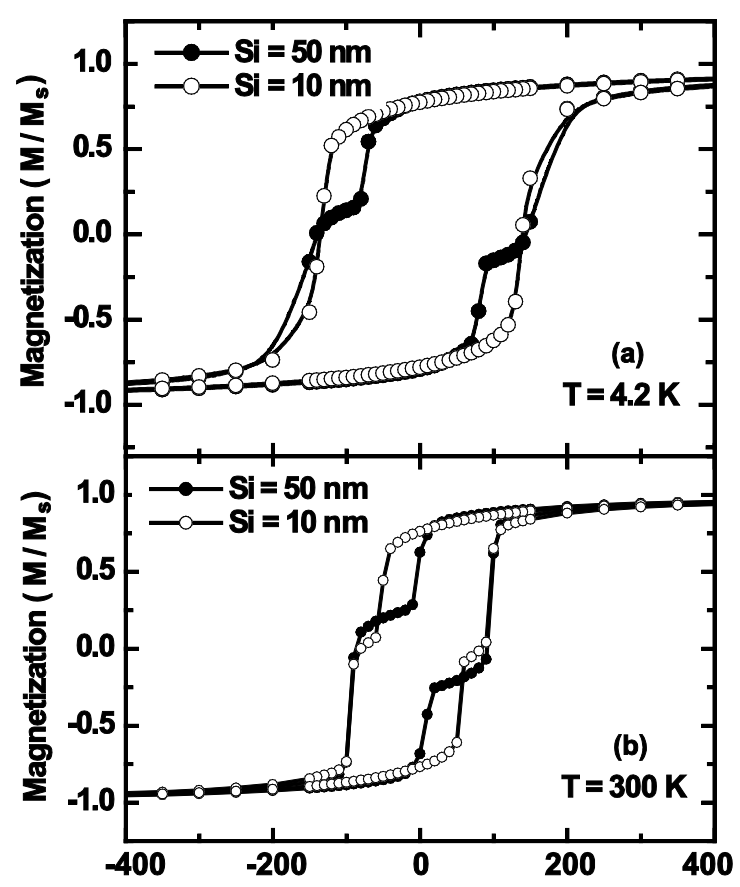

Fig. 1. Magnetization processes of $\mathrm{Co} / \mathrm{Si} / \mathrm{Co} / \mathrm{GaAs}$ multilayer without buffer layer at (a) $4.2 \mathrm{~K}$ and (b) $300 \mathrm{~K}$, for different values of Si interlayer thickness.

For $d_{S i}$ less than or equal $10 \mathrm{~nm}$, two Co layers were magnetically coupled to each other and the resultant hysteresis loop became single phase. But it is observed that the coupling strength between two magnetic Co layers is decreased as the thickness of Si interlayer, $d_{S i}$, is increased from $10 \mathrm{~nm}$ to $25 \mathrm{~nm}$. An antiparallel spin state exists in the multilayer when the thickness of $\mathrm{Si}$ interlayer became $50 \mathrm{~nm}$. The hysteresis loop of the same sample at $300 \mathrm{~K}$ is shown in Fig. 1(b). It is seen from the figure that the magnetic coupling between the soft and hard Co layers is decreased at room temperature. Twophase hysteresis loop is observed at both the Si interlayer thicknesses. The coercive fields 
of both the soft and hard magnetic layers are decreased. This is due to the fact that the antiparallel spin state became gradually weaker between ferromagnetic layers with the increase of $\mathrm{Si}$ interlayer thickness. For $10 \mathrm{~nm}$ Si spacer, the coupling between the ferromagnetic layers is decreased and multilayer showed two-phase hysteresis loop at $300 \mathrm{~K}$. This might be the spin fluctuations in ferromagnetic later at that temperature. Although the mechanism of antiferromagnetic coupling in metal/metal multilayer is wellestablished, it is still not clear how the mechanism can be modified for coupling across semiconducting spacer layer. However, without a buffer layer a significant chemical intermixing occurs between ferromagnetic metal and semiconductor substrate which influences the magnetization process of the multilayer [11]. To investigate the effect of buffer layer on magnetization process of the multilayer, $1 \mathrm{~nm}$ buffer layer of $\mathrm{Au}$ is deposited between Co and Si spacer. Magnetization process of the multilayer $\left(d_{S i}=50\right.$ $\mathrm{nm}$ ) with Au buffer layer is shown in Fig. 2. Two-phase hysteresis loop is completely disappeared and became single-phase with Au buffer layer at $4.2 \mathrm{~K}$. Two-phase hysteresis loop is reappeared at $300 \mathrm{~K}$ with buffer layer.

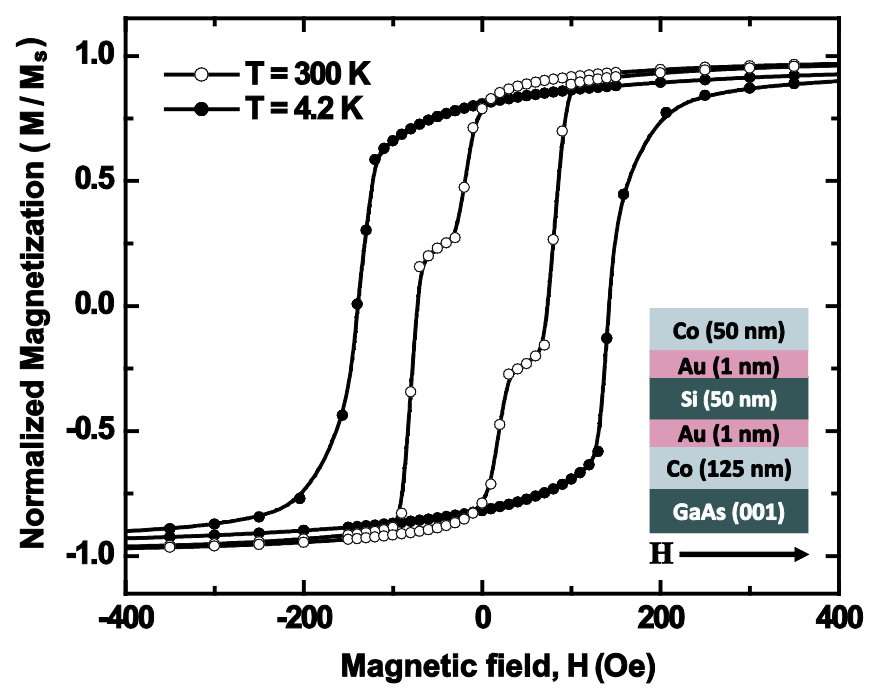

Fig. 2. Magnetization processes of $\mathrm{Co} / \mathrm{Au} / \mathrm{Si} / \mathrm{Au} / \mathrm{Co} / \mathrm{GaAs}$ multilayer. The applied magnetic field was parallel to the film plane.

Two-phase hysteresis loop found in the multilayer without buffer layer could be better quantified by measuring $\mathrm{Si}$ interlayer thickness dependent coercivity at various temperatures. The coercivity of $50 \mathrm{~nm}$ Co layer in the multilayer varies with Si interlayer thickness. Si interlayer thickness-dependent coercivity of Co layers is shown in Fig. 3. 


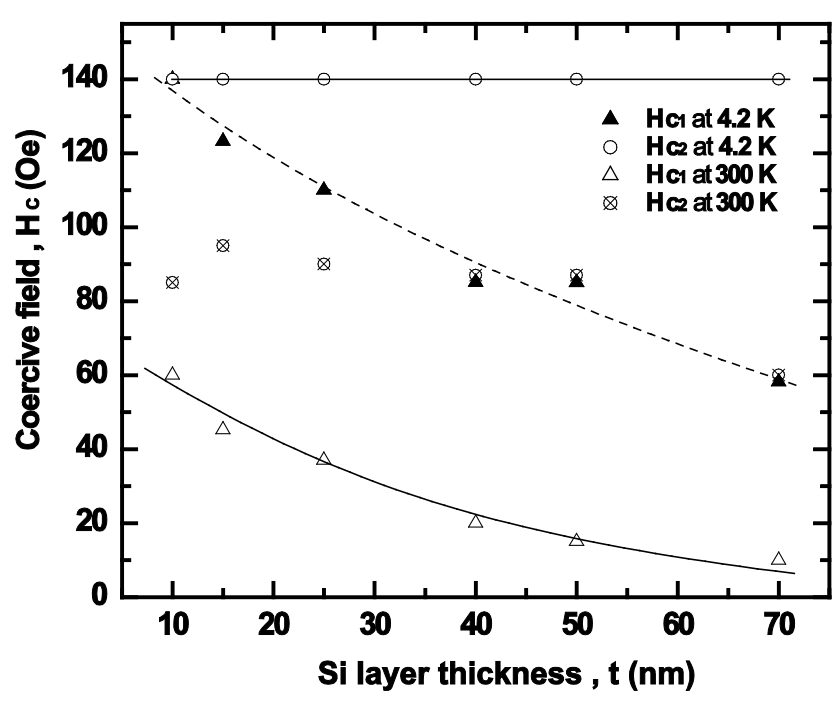

Fig. 3. Si interlayer thickness dependent coercive field of Co layers in the multilayer without buffer layer. The direction of the applied magnetic field was parallel to the film plane.

At thinner Si-spacer layer $\left(d_{S i}<=10 \mathrm{~nm}\right)$, coercivities of $50 \mathrm{~nm}$ Co $\left(H_{\mathrm{C} 1}\right)$ and $125 \mathrm{~nm}$ Co $\left(H_{\mathrm{C} 2}\right)$ layers were comparable to each other at $4.2 \mathrm{~K}$. Therefore, the hysteresis loop was single-phase. But the values of $H_{\mathrm{C} 1}$ and $H_{\mathrm{C} 2}$ were different at $300 \mathrm{~K}$. Thus the multilayer showed two-phase hysteresis loop with $d_{S i}$ equal to $10 \mathrm{~nm}$ at $300 \mathrm{~K}$. On the other hand, the difference in coercivity between soft and hard Co layers increases gradually with the increase of Si interlayer thickness. Therefore the multilayer having $d_{S i}=>15 \mathrm{~nm}$ showed two-phase hysteresis loop in the temperature range 4.2-300 K.

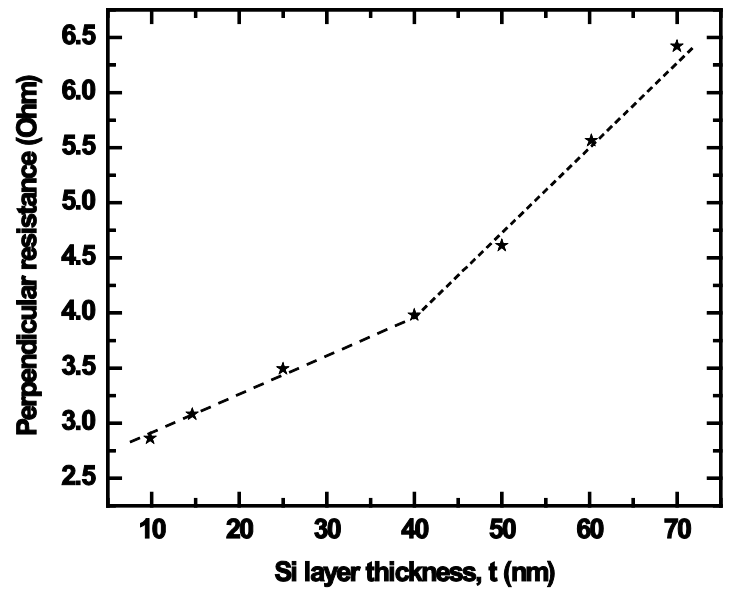

Fig 4. Perpendicular resistance vs. Si interlayer thickness of $\mathrm{Co} / \mathrm{Si} / \mathrm{Co} / \mathrm{GaAs}$ multilayer without buffer layer. 
Si interlayer thickness dependent perpendicular resistance of the multilayer is shown in Fig. 4. It is observed that perpendicular resistance of the multilayer increases with the increase of Si interlayer thickness. The curve shows two slopes. The slopes of the curve can be explained with help of interlayer mixing and silicides formation the interface between Co and Si without buffer layer.

Cobalt silicides phase is formed at the interface of Co and Si [5-7,16]. The electrical conductivity of cobalt silicides is much higher than that of Si interlayer [17]. Therefore, the two slopes found in the resistance vs. Si interlayer thickness curve can be explained with the help of silicides formation at the interface. The effective resistance of the multilayer was low at thinner Si interlayer thickness $(10-40 \mathrm{~nm})$ because the thickness of cobalt silicides was comparable with Si interlayer thickness. However, the thickness of the interface (cobalt silicides) is limited to a certain extent in the Si layer. The effect of cobalt silicides on the total resistance of the multilayer became less dominant as the thickness of the $\mathrm{Si}$ interlayer is increased $\left(d_{S i}>40 \mathrm{~nm}\right)$. We suggest that two-phase hysteresis loop observed in the multilayer $\left(d_{S i}=50 \mathrm{~nm}\right)$ without buffer layer was due to the formation of cobalt silicides since it did not appear in the multilayer with Au buffer layer at $4.2 \mathrm{~K}$. Two-phase hysteresis loop was observed at $300 \mathrm{~K}$ in the multilayer with Au buffer layer. This might be the spin fluctuations in ferromagnetic layer at that temperature. We propose that formation of cobalt silicides at the interface of $\mathrm{Co}$ and $\mathrm{Si}$ modulate magnetic properties of the multilayer without buffer layer.

\section{Conclusion}

The coercivity of $50 \mathrm{~nm}$ Co decreased with increasing $\mathrm{Si}$ interlayer thickness. Perpendicular resistance of the multilayer increased with increasing Si interlayer thickness. Two-phase hysteresis loop was observed in the multilayer with $d_{S i}=>15 \mathrm{~nm}$ without buffer layer. Two-phase hysteresis loop became single-phase with $d_{S i}=50 \mathrm{~nm}$ with Au buffer layer at $4.2 \mathrm{~K}$. Phase transformation in the multilayer indicated that certain electronic properties of cobalt silicides might modulate magnetic properties of the multilayer without buffer layer.

\section{Acknowledgements}

This work was partially supported by finance from the Ministry of Education, Culture, Sports, Science and Technology of the Japanese Government.

\section{References}

1. A. Wolf, D. D. Awschalom, R. A. Buhrman, J. M. Daughton, S. von Molnár, M. L. Roukes, A. Y. Chtchelkanova, and D. M. Treger, Science 294, 1488 (2001). PMid:11711666

2. G. A. Prinz, Science 282, 1660 (1998). http://dx.doi.org/10.1126/science.282.5394.1660

3. G. A. Prinz, J. Magn. Magn. Mater. 200, 57 (1999). http://dx.doi.org/10.1016/S0304-8853(99)00335-2

4. S. Agrawal, M. B. A. Jalil, and K. L. Teo, J. Appl. Phys. 97, 103907 (2005). 
http://dx.doi.org/10.1063/1.1886886

5. S. Abhaya, G. Venugopal Rao, S. Kalavathi, V. S. Sastry, and G. Amarendra, Surf. Sci. 600, 2762 (2006). http://dx.doi.org/10.1016/j.susc.2006.04.043

6. K. Inomata and Y. Saito, J. Appl. Phys. 81, 5344 (1997). http://dx.doi.org/10.1063/1.364540

7. M. Diale, C. Challens, and E. C. Zingu, Appl. Phys. Lett. 62, 943 (1993). http://dx.doi.org/10.1063/1.108527

8. G. S. Patrin, Chan-Gyu Lee, Bon-Heun Koo, and Keesam Shin, Phys. Lett. A 359, 149 (2006). http://dx.doi.org/10.1016/j.physleta.2006.06.017

9. H. A. M. van de Berg, W. Clemens, G. Giers, G. Rupp, W. Schelter, and M. Vieth, IEEE Trans. Magn. 32, 4624 (1996). http://dx.doi.org/10.1109/20.539099

10. E. E. Fullerton, D. T. Margulies, M. E. Schabers, M. Carey, B. Gurney, A. Moser, M. Best, G. Zeltzer, K. Rubin, and H. Rosen, Appl. Phys. Letters 77, 3806 (2000). http://dx.doi.org/10.1063/1.1329868

11. L. M. Falicov, D. T. Pierce, S. D. Bader, R. Gronsky, K.B. Hathaway, H. J. Hopster, D. N. Lambeth, S. P. Parkin, G. Prinz, M. Salamon, I. K. Schuller, and R. H. Victora, J. Mater. Res. 5, 1299 (1990). http://dx.doi.org/10.1557/JMR.1990.1299

12. B. Briner and M. Landolt, Phys. Rev. Lett. 73, 340 (1994). http://dx.doi.org/10.1103/PhysRevLett.73.340

13. G. S. Patrin, N. V. Volkov, S. G. Ovchinnikov, E. V. Eremin, M. A. Panova, and S. N. Varnakov, JETP Letters 80, 491 (2004). http://dx.doi.org/10.1134/1.1839297

14. S. A. Haque, A. Matsuo, Y. Yamamoto, and H. Hori, J. Magn. Magn. Mater. 257, 313 (2003). http://dx.doi.org/10.1016/S0304-8853(02)01228-3

15. J. Islam, Y. Yamamoto, and H. Hori, J. Magn. Magn. Mater. 310, 2234 (2007). http://dx.doi.org/10.1016/j.jmmm.2006.10.815

16. S. Roy and B. N. Dev, Phys. Status Solidi (a) 209, 1511(2012). http://dx.doi.org/10.1002/pssa.201127765

17. Bing-Zong Li , P. Liu , G. B. Jiang, W. N. Huang, X. Lu, R. G. Aitken, K. Daneshvar, and M. Puzerewski, and G. Singco, J. Appl. Phys. 70, 5427 (1991). http://dx.doi.org/10.1063/1.350200 